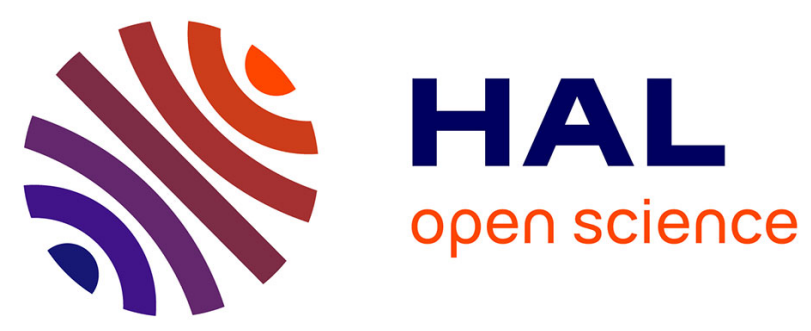

\title{
Unifying Approaches and Removing Unrealistic Assumptions in Shape from Shading: Mathematics Can Help
}

Emmanuel Prados, Olivier Faugeras

\section{- To cite this version:}

Emmanuel Prados, Olivier Faugeras. Unifying Approaches and Removing Unrealistic Assumptions in Shape from Shading: Mathematics Can Help. Proceedings of the 8th European Conference on Computer Vision, May 2004, Prague, Czech Republic, European Union. pp.141-154, 10.1007/b97873 . inria-00394234

\section{HAL Id: inria-00394234 \\ https://hal.inria.fr/inria-00394234}

Submitted on 11 Jun 2009

HAL is a multi-disciplinary open access archive for the deposit and dissemination of scientific research documents, whether they are published or not. The documents may come from teaching and research institutions in France or abroad, or from public or private research centers.
L'archive ouverte pluridisciplinaire HAL, est destinée au dépôt et à la diffusion de documents scientifiques de niveau recherche, publiés ou non, émanant des établissements d'enseignement et de recherche français ou étrangers, des laboratoires publics ou privés. 


\title{
Unifying approaches and removing unrealistic assumptions in Shape From Shading: Mathematics can help
}

\author{
Emmanuel Prados and Olivier Faugeras \\ Odyssée Lab., INRIA/ENS/ENPC, France. \\ http://www-sop.inria.fr/odyssee/
}

\begin{abstract}
This article proposes a solution of the Lambertian Shape From Shading (SFS) problem by designing a new mathematical framework based on the notion of viscosity solutions. The power of our approach is twofolds: 1) it defines a notion of weak solutions (in the viscosity sense) which does not necessarily require boundary data. Note that, in the previous SFS work of Rouy et al. [23,15], Falcone et al. [8], Prados et al. $[22,20]$, the characterization of a viscosity solution and its computation require the knowledge of its values on the boundary of the image. This was quite unrealistic because in practice such values are not known. 2) it unifies the work of Rouy et al. [23, 15], Falcone et al. [8], Prados et al. $[22,20]$, based on the notion of viscosity solutions and the work of Dupuis and Oliensis [6] dealing with classical $\left(C^{1}\right)$ solutions. Also, we generalize their work to the "perspective SFS" problem recently introduced by Prados and Faugeras [20].

Moreover this article introduces a "generic" formulation of the SFS problem. This "generic" formulation summarizes various (classical) formulations of the Lambertian SFS problem. In particular it unifies the orthographic and the perspective SFS problems. This "generic" formulation significantly simplifies the formalism of the problem. Thanks to this generic formulation, a single algorithm can be used to compute numerical solutions of all these previous SFS formulations.

Finally we propose two algorithms which provide numerical approximations of the new weak solutions of the "generic SFS" problem. These provably convergent algorithms are quite robust and do not necessarily require boundary data.
\end{abstract}

\section{Introduction}

The application of the theory of Partial Differential Equations (PDEs) to the Shape from Shading (SFS) problem has been hampered by several types of difficulties. The first type arises from the kind of modelling that is used: orthographic cameras looking at Lambertian objects with a single point light source at infinity is the set of usual assumptions [29,10]. The second type is mathematical: characterizing the solution(s) of the corresponding PDE has turned out to be a very difficult problem; boundary conditions are assumed to be known, say at 
image boundary, in contradiction with real practice $[23,22,8]$. The third type is algorithmic: assuming that existence has been proved, coming up with provably convergent numerical schemes has turned out to be quite involved [7].

Our approach is therefore based upon the interaction of the following three areas:

1. Mathematics: We use and "extend" the notion of viscosity solutions to solve such basic problems as the existence and uniqueness of a solution or the characterization of all solutions when uniqueness does not hold.

2. Algorithmic: In [2], Barles and Souganidis propose a large class of approximation schemes (called monotonous) of these solutions. Inspired by their work, we build such schemes for the SFS equations from which we obtain algorithms whose properties we can analyze in detail (stability, convergence, accuracy). This results in provably correct code within a set of well-defined assumptions.

3. Modeling: The classical theory of viscosity solutions (used until now for solving the SFS problem $[23,15,22,20,8])$ is not well-adapted to the natural constraints of the SFS problem. In particular it requires that boundary conditions be given, e.g. at the image boundary, and creates undesirable folds (see section 3). In order to be able to get rid of this constraint, we have adapted the notion of viscosity solutions.

Our contributions are first in the area of Mathematics: we adapt the notion of singular viscosity solutions (recently developed by Camilli and Siconolfi $[3,4]$ ) for obtaining a "new" class of viscosity solutions which is really more suitable to the SFS problem than the previous ones. This mathematical framework is very general and allows to improve and unify the work of $[23,15,6,22,20,8]$. Directly connected to the area of modeling, thanks to the introduction of this framework, we are able to relax the very constraining assumption that boundary conditions are known. Concerning the area of modeling, we extend the work of [20]: considering a pinhole camera, we allow the light source to be either at infinity or approximately at the optical center, as in the case of a flash. We also show that the orthographic and pinhole camera SFS equations are special cases of a general equation, thereby simplifying the formalization of the problem. Our contributions are also algorithmic: we propose two provably convergent approximation schemes for our "generic" SFS equation. Moreover, one of the algorithms we propose seems to be the most efficient iterative algorithms of the SFS literature. The article is written in a non mathematical style. The reader interested in the proofs is referred to $[19,21]$.

\section{A unification of the "perspective" and "orthographic SFS"}

We deal with Lambertian scenes and suppose that the albedo is constant and equal to 1 . The scene is represented by a surface $S$. Let $\Omega$, the image, be the rectangular domain $] 0, X[\times] 0, Y[. S$ can be explicitly parameterized by using 
a function defined on the closure $\bar{\Omega}$. The particular type of parametrization is irrelevant here but may vary according to the camera type (orthographic versus pinhole) and the position of light source (finite or infinite distance). We note $I$ the image intensity, a function from $\bar{\Omega}$ into the closed interval $[0,1]$. The Lambertian hypothesis implies:

$$
I(x)=\frac{\mathbf{n}(x) \cdot \mathbf{L}}{|\mathbf{n}(x)|},
$$

where $\mathbf{n}(x)$ is a normal vector to the surface $S$ at the point $S(x)$ and $\mathbf{L}$ is the unit vector representing the light direction at this same point (the light source is assumed to be a point). Despite the notation, $\mathbf{L}$ can depend on $S(x)$, if the point source is at a finite distance from the scene.

\section{1 "Orthographic SFS" with a point light source at infinity}

This is the traditional setup for the SFS problem. We denote by $\mathbf{L}=(\alpha, \beta, \gamma)$ the unit vector representing the direction of the light source $(\gamma>0), \mathbf{l}=(\alpha, \beta)$, and $u$ the distance of the points in the scene to the camera. The SFS problem is then, given $I$ and $\mathbf{L}$, to find a function $u: \bar{\Omega} \longrightarrow \mathbb{R}$ satisfying the brightness equation:

$$
\forall x \in \Omega, \quad I(x)=(-\nabla u(x) \cdot \mathbf{l}+\gamma) / \sqrt{1+|\nabla u(x)|^{2}},
$$

In the SFS literature, this equation is rewritten in a variety of ways as $H(x, p)=$ 0 , where $p=\nabla u$ :

1) In [23], Rouy and Tourin introduce $H_{R / T}(x, p)=I(x) \sqrt{1+|p|^{2}}+p \cdot \mathbf{l}-\gamma$.

2) In [6], Dupuis and Oliensis consider

$$
H_{D / O}(x, p)=I(x) \sqrt{1+|p|^{2}-2 p \cdot \mathbf{l}}+p \cdot \mathbf{l}-1 .
$$

(use the change of variables: $\Psi\left(x_{1}, x_{2}, z\right)=\left(x_{1}, x_{2}, x_{1} \alpha+x_{2} \beta+z \gamma\right)$ )

$3)$ In the case where $\mathbf{L}=(0,0,1)$, Lions et al. [15] deal with:

$$
H_{\text {Eiko }}(x, p)=|p|-\sqrt{\frac{1}{I(x)^{2}}-1} . \quad \text { (called the Eikonal equation) }
$$

The function $H$ is called the Hamiltonian.

\section{2 "Perspective SFS" with a point light source at infinity}

Few SFS approaches deal with the perspective projection problem. To our knowledge, only eight authors $[17,13,9,27,28,20,26,5]$ consider a pinhole camera model instead of an affine or orthographic model. Among these papers, only the work of Prados and Faugeras [20] proposes a formalism completely based on Partial Differential Equations (PDEs) and provides a rigourous mathematical study. The camera is characterized and represented by the retinal plane $R$ and by the optical center as shown in figure 1 . We note $f$ the focal length. We assume that $S$ can be explicitly parameterized by the depth modulation function $u$ defined on $\bar{\Omega}$ :

$$
S=\{u(x) .(x,-f) ; \quad x \in \bar{\Omega}\},
$$

and that the surface is visible (in front of the retinal plane) hence $u \geq 1$.

We also note $\mathbf{L}=(\alpha, \beta, \gamma)$ the unit vector representing the direction of the light source $(\gamma>0)$. Combining the expression of $\mathbf{n}(x)$ (easily obtained through 
differential calculus) and the change of variables $v=\ln (u)$, Prados and Faugeras $[18,20]$ obtain from the irradiance equation the following Hamiltonian:

$$
H_{P / F}(x, p)=I(x) \sqrt{f^{2}|p|^{2}+(x \cdot p+1)^{2}}-(f \mathbf{l}+\gamma x) \cdot p-\gamma ;
$$

By using the change of variables $v(x)=\frac{\gamma}{f}[\gamma f-\mathbf{l} \cdot x] u(x)$, we obtain another Hamiltonian $H_{P e r s}(x, p)$ which verifies more interesting properties (see [19]).

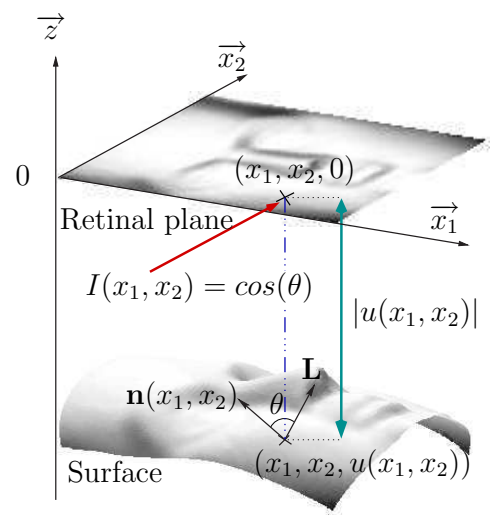

a) orthogonal projection

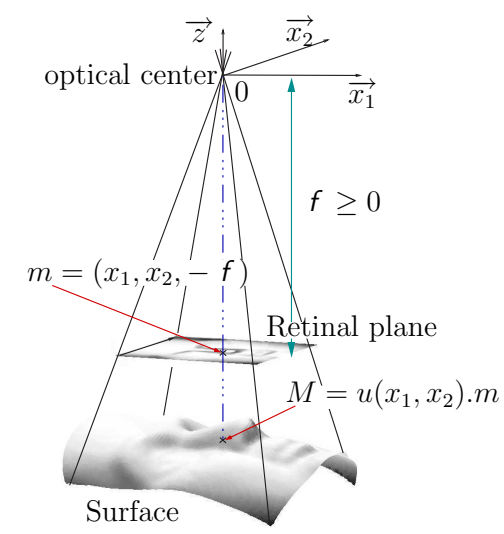

b) perspective projection

Fig. 1. Images arising from an orthogonal (versus perspective) projection.

\section{3 "Perspective SFS" with a single point light source located at the optical center}

We present a new formulation of the "perspective SFS". This approximately models the situation encountered when we use a simple camera equiped with a flash and the scene is relatively far from the camera. In this case, we represent the scene by the surface $S$ defined by $S=\left\{\frac{f u(x)}{\sqrt{|x|^{2}+f^{2}}}(x, f) ; \quad x \in \bar{\Omega}\right\}$. Using the same trick as in the previous section $(v=\ln (u))$, we readily obtain the Hamiltonian:

$$
H_{F}(x, p)=I(x) \sqrt{f^{2}|p|^{2}+(p \cdot x)^{2}+Q(x)^{2}}-Q(x),
$$

where $Q(x)=\sqrt{f^{2} /\left(|x|^{2}+f^{2}\right)}$. See [19] for more details.

\subsection{A generic Hamiltonian}

In [19], we prove that all the previous SFS Hamiltonians are special cases of the following "generic" Hamiltonian:

$$
H_{g}(x, p)=\tilde{H}_{g}\left(x, A_{x} p+\overrightarrow{v_{x}}\right)+\overrightarrow{w_{x}} \cdot p+c_{x}
$$


with $\quad \tilde{H}_{g}(x, q)=\kappa_{x} \sqrt{|q|^{2}+K_{x}^{2}}$, $\kappa_{x}, K_{x} \geq 0, A_{x}=D_{x} R_{x}, D_{x}=\begin{array}{cc}\mu_{x} & 0 \\ 0 & \nu_{x}\end{array}, R_{x}$ is the rotation matrix $\frac{1}{|x|} \begin{array}{cc}x_{2} & -x_{1} \\ x_{1} & x_{2}\end{array}$ if $x \neq 0, R_{x}=I d_{2}$ if $x=0, \mu_{x}, \nu_{x} \neq 0\left(\mu_{x}, \nu_{x} \in \mathbb{R}\right), \overrightarrow{v_{x}}, \overrightarrow{w_{x}} \in \mathbb{R}^{2}$ and $c_{x} \in \mathbb{R}$.

By using the Legendre transform, we rewrite this Hamiltonian as a "generic" Hamilton-Jacobi-Bellman (HJB) Hamiltonian:

$$
H_{g}(x, p)=\sup _{a \in B_{2}(0,1)}\left\{-f_{g}(x, a) \cdot p-l_{g}(x, a)\right\} .
$$

In [19], we detail the exact expressions of $f_{g}$ and $l_{g}$. The HJB formulations of the Hamiltonians $H_{E i k o}, H_{D / O}, H_{R / T}$ and $H_{P / F}$, respectively given in $[23,6,22$, 20], are special cases of the above generic formulation; thereby ours is a generalization and a unification of these works. This generic formulation considerably simplifies the formalism of the problem. All theorems about the characterization and the approximation of the solution are now proved by using this generic SFS Hamiltonian. In particular, this formulation unifies the orthographic and perspective SFS problems. Also, from a practical point of view, a unique code can be used to numerically solve these two problems.

\section{Weaknesses of the previous theoretical approaches}

The notion of viscosity solutions was first used to solve SFS problems by Lions, Rouy and Tourin $[23,15]$ in the 90s. Their work was based upon the notion of continuous viscosity solution. The viscosity solutions are PDE solutions in a weak sense. In particular, they are not necessarily differentiable and can have edges. This notion allows to define a solution of a PDE which does not have classical solutions. For example, the equation

$$
|\nabla u(x)|=1 \text { for all } x \text { in }] 0,1[
$$

with $u(0)=u(1)=0$, does not have classical solutions (Rolles theorem) but has a continuous viscosity solution (see figure 2-a)). Let us emphasize that continuous viscosity solutions are continuous (on the closure of the set where it is defined) and that a solution in the classical sense is a viscosity solution. The weakness of this notion is due to the compatibility condition necessary to the existence of the solution (constraint on the variation of the boundary conditions [14]). Also, the same equation (2) with $u(0)=0, u(1)=1.5$ does not have continuous viscosity solutions. Now let us suppose that we make a large error

a)

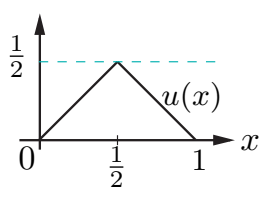

b)

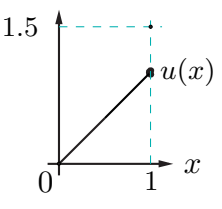

Fig. 2.

a) Continuous viscosity solution of (2) with $u(0)=u(1)=0$;

b) discontinuous viscosity solution of (2) with $u(0)=0$ and $u(1)=1.5$. 
on the boundary condition, when we compute a numerical solution of the SFS problems. If this error is too large then there do not exist continuous viscosity solutions. In this case one may wonder what the numerical algorithm of $[23,15]$ computes. In [22], Prados et al. answer this question by proposing to use the more general idea of discontinuous viscosity solutions. For example, equation (2) with $u(0)=0, u(1)=1.5$ has a discontinuous viscosity solution (see figure 2 -b)). Let us emphasize that a "discontinuous viscosity solution" can have discontinuities and that a continuous viscosity solution is a discontinuous viscosity solution.

The classical theory of viscosity solutions offers simple and general theorems of existence and uniqueness of solutions for exactly the type of PDEs that arise in the context of SFS. In particular the theory allows to characterize exactly all possible continuous viscosity solutions: given a particular Dirichlet condition on the image boundary (verifying the compatibility condition), if the set of critical points (points of maximal intensity, i.e. $I(x)=1$ ) is empty, then there exists a unique continuous viscosity solution satisfying the boundary conditions; If the set of critical points is not empty there exists an infinity of continuous solutions which are characterized by their values at the critical points. Note that this result is general and applies equally to all the SFS models described in section 2 (see [19]). As a consequence, the SFS problem is ill-posed and to compute an approximation of a solution, Rouy et al. and Prados et al. [23, 22, 20] must assume that the values of the solutions are given at the image boundary and the critical points. This is quite unsatisfactory, even more so since small errors on these values create undesirable crests, see figure 3-b) or [22] for an example

a)

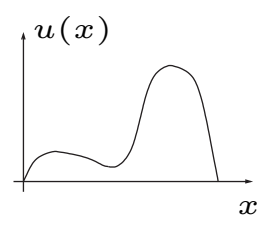

b)

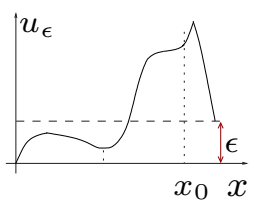

c)

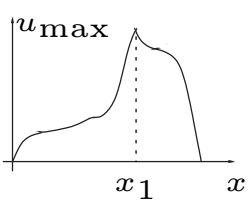

Fig. 3. a) original surface $u$; b) solution $u_{\epsilon}$ associated to corrupted boundary conditions and to the image obtained from the original surface a) with the Eikonal equation; c) maximal solution $u_{\max }$ (in Falcone's sense [8]) associated to the same image. $u_{\epsilon}$ and $u_{\text {max }}$ present a kink at $x_{0}$ and $x_{1}$.

with a real image. Falcone [8] proposes not to specify anymore the values of the solution at the critical points (he still requires to specify the values at the image boundary though). In order to achieve this, he uses the notion of maximal viscosity solutions developed by Camilli and Siconolfi [3]. Despite its advantages, this approach is not really adapted to the SFS problem, see for example figure 3 -c). In this figure, the maximal solution $u_{\max }$ associated to the image obtained from the original surface $u$ shows a highly visible crest where the surface should be smooth. Even with the correct boundary conditions, Falcone's method does not really provide a suitable solution. 
To summarize, the work of Rouy et al. [23], Prados et al. [22, 20] and Falcone et al. [8] suggests theories and numerical methods based on the concept of viscosity solutions and requiring data on the boundary of the image. At the opposite, Dupuis and Oliensis [6] consider $C^{1}$ solutions. They characterize a $C^{1}$ solution by specifying only its values at the critical points which are local minima. In particular, they do not specify the values of the solution on the boundary of the image. Also, they provide algorithms for approximating these smooth solutions. Nevertheless, in practice, because of noise, of incorrect modelization, errors on parameters or on the depth values enforced at the critical points, there do not exist $C^{1}$ solutions to the SFS equations [16]. Therefore, the theory of Dupuis and Oliensis does not apply.

Considering the drawbacks and the advantages of all these methods, it seems important to define a new class of weak solutions such that the characterization of Dupuis and Oliensis holds, and which provides a (theoretical and numerical) solution when there do not exist smooth solutions.

As we show in [21], the classical notion of viscosity solutions, like the notion of singular viscosity solutions (pioneered by Ishii and Ramaswamy [11] and recently upgraded by Camilli and Siconolfi [3]) does not provide a direct extension of the Dupuis and Oliensis work. For such an extension, we must modify these notions and we must consider a "new" type of boundary conditions (called "state constraints" [24]). It turns out that the correct notion of viscosity solution for the SFS problem is the "singular discontinuous viscosity solution with Dirichlet boundary conditions and state constraints". These solutions can be interpreted as maximal solutions and have the great advantage of not necessarily requiring boundary or critical points conditions. Moreover, this notion provides a mathematical framework unifying the work of Rouy et al. [15, 23], Prados et al. [22, 20], Falcone et al. [8] and Dupuis and Oliensis [6].

\section{Singular discontinuous viscosity solutions for SFS}

In this section we briefly describe the notion of "singular discontinuous viscosity solutions with Dirichlet boundary conditions and state constraints" (SDVS). We refer to [21] for more details. Also we do not recall the classical definition of viscosity solutions: see [1] for a recent overview.

Considering the generic SFS problem, we concentrate on the following HJB equation:

$$
\sup _{a \in A}\{f(x, a) \cdot \nabla u(x)-l(x, a)\}=0, \quad \forall x \in \Omega
$$

To simplify, we assume in this paper ${ }^{1}$ that $l \geq 0$ and we denote $\mathcal{S}:=\{x \mid l(x, a)=$ 0 for some $a \in A\}$. Also assume that $\mathcal{S}$ verifies $\mathcal{S} \cap \partial \Omega=\emptyset$. To equation

\footnotetext{
${ }^{1}$ In [21], we do not assume that $l \geq 0$. Also, the definition of $\mathcal{S}$ and the developed tools are more sophisticated. Note that, except for $H_{R / T}$ and $H_{P / F}$, all the SFS Hamiltonians verify $l \geq 0$. This justifies our interest for the Hamiltonian $H_{D / O}$ and the original one $H_{\text {Pers }}$.
} 
(3), we add Dirichlet boundary conditions (DBC) on the boundary of the image and on $\mathcal{S}$ :

$$
u(x)=\varphi(x), \quad \forall x \in \partial \Omega \cup \mathcal{S} ;
$$

$\varphi$ being continuous on $\partial \Omega \cup \mathcal{S}$ into $\mathbb{R} \cup\{+\infty\}$ (but $\varphi \neq+\infty$ everywhere). At the points $x$ s.t. $\varphi(x)=+\infty$, we say that we impose a state constraint [24]. In the SFS context, $\mathcal{S}$ is the set of critical points $\{x \mid I(x)=1\}$.

Definition: $u$ is a SDVS of (3)-(4), if $u$ is a discontinuous viscosity solution of (3)-(4) on $\bar{\Omega}-\mathcal{S}$ and if $\forall x \in \mathcal{S},\left[u^{*}(x) \leq \varphi(x)\right]$ and $\left[u_{*}(x) \geq \varphi(x)\right.$ or $u_{*}$ is a singular viscosity supersolution in Camilli's sense at the point $x$ ].

Definitions of $u_{*}$ and $u^{*}$ (not detailed here because of space) can be found in [1]. The notion of singular viscosity supersolution in Camilli's sense is completely described in $[3,4]$.

In [21], we prove the existence and the uniquess of the SDVS of all SFS equations as soon as $I$ is Lipschitz continuous and the Hamiltonian is coercive (e.g. $H_{D / O}$ and $H_{R / T}$ are coercive $\left.\Leftrightarrow I(x)>|\mathbf{1}|\right)$. We also prove the robustness of this solution to pixel noise and to errors on the light or focal length parameters. Finally, note that, when we impose state contraints on the boundary of the images and some critical points, this solution can be interpreted as the maximal viscosity solution. See [21] for more details.

\section{A general framework for SFS:}

The main interest of this "new" class of solutions lies in the possibility to impose the heights of the solution at the critical points when we know them (this is impossible with discontinuous viscosity solutions; it is possible with continuous viscosity solutions but compatibility conditions are required) and in the possibility to "send at infinity" the boundary conditions when we do not know then (this possibility is not considered by Falcone et al. [8]). The relevance of this notion is amplified by its consistency with the work of Dupuis and Oliensis [6]. This is illustrated by the following proposition (see [21]):

Proposition 1 Let $u$ be a $C^{1}$ solution of equation (3). Let $\tilde{\mathcal{S}}$ be the subset of $\mathcal{S}$ corresponding to the local minima of $u$. If u verifies the assumption 2.1 of $[6]^{2}$ then $u$ is the SDVS of (3)-(4) for $\varphi(x)=u(x) \forall x \in \tilde{\mathcal{S}}$ and $\varphi(x)=+\infty$ elsewhere.

Therefore, when there do not exist $C^{1}$ solutions, the SDVS consistently extend the work of Dupuis and Oliensis. Moreover, the SDVS unify the various theories used for solving the SFS problem. In effect, we can verify that [21]:

- In the case where the $\mathrm{DBC}$ are finite on $\partial \Omega \cup \mathcal{S}$ and the compatibility condition (see [14]) holds, then the SDVS of (3)-(4) is the continuous viscosity solution used by $[23,15,22,20]$.

\footnotetext{
${ }^{2}$ Not stated here because of space.
} 
- When the DBC are finite on the boundary of the image and state constraints are imposed at the critical points, the SDVS of (3)-(4) corresponds to Camilli's singular viscosity solutions $[3,4]$ used by Falcone [8].

- As seen above, the SDVS corresponds to the $C^{1}$ solution of (3), verifying the assumption 2.1 of Dupuis and Oliensis [6].

Consequently, the theoretical results of Falcone et al. [8] Rouy et al. [23, 15], Prados et al. [22,20] and Dupuis et al. [6] are automatically extended to the "perspective $S F S$ " (use $H_{F}$ and $H_{\text {pers }}$ ). Finally, one can conjecture that by using the work of $[12,25]$, the notion of SDVS can be extended to solve SFS problem with discontinuous images. This would be very difficult without the tool of viscosity solutions.

\section{Numerical approximation of the SDVS for generic SFS}

This section explains how to compute a numerical approximation of the SDVS of the generic SFS equation. This requires three steps. First we "regularize" the equation. Second, we approximate the "regularized" SFS equation by approximation schemes. Finally, from the approximation schemes, we design numerical algorithms.

\section{Regularisation of the generic SFS equation:}

For an intensity image $I$ and $\epsilon>0$, let us consider the truncated image $I_{\epsilon}$ defined by $I_{\epsilon}(x)=\min (I(x), 1-\epsilon)$. By using a stability result, we prove that for the generic SFS Hamiltonian, the SDVS associated with the image $I_{\epsilon}$ converges uniformly toward the SDVS associated with the image $I$, when $\epsilon \rightarrow 0$. Also, $\forall \epsilon>0$, the generic SFS equation associated with $I_{\epsilon}$ is no more degenerate. Thus for approximating this equation, we can use the classical tools developed by Barles and Souganidis [2].

\section{Approximation schemes for the nondegenerate SFS equations:}

Let us consider the "regularized" generic SFS equation. The theory of Barles and Souganidis [2] suggests to consider monotonous schemes. Therefore, we construct the following monotonous scheme (we call it "implicit") $S(\rho, x, u(x), u)=0$ with

$$
S(\rho, x, t, u)=\max _{s_{1}, s_{2}= \pm 1} S_{s_{1}, s_{2}}(\rho, x, t, u),
$$

where $\rho=\left(\Delta x_{1}, \Delta x_{2}\right)$ is the mesh size and where we choose:

$$
S_{s_{1}, s_{2}}^{i m p l}(\rho, x, t, u)=\sup _{a \in A_{s_{1}, s_{2}}}\left\{-f_{g}(x, a) \cdot\left(\frac{\left.\frac{t-u\left(x+s_{1} \Delta x_{1} \overrightarrow{1_{1}}\right)}{-s_{1} \Delta x_{1} \vec{x}_{1}}\right)}{\frac{t-u\left(x+s_{2} \Delta x_{2} \overrightarrow{2_{2}}\right)}{-s_{2} \Delta x_{2}}}\right)-l_{g}(x, a)\right\} .
$$

$A_{s_{1}, s_{2}}=\left\{a \in A \mid f_{g_{1}}(x, a) s_{1} \geq 0\right.$ and $\left.f_{g_{2}}(x, a) s_{2} \geq 0\right\}$.

By introducing a fictitious time $\Delta \tau$, we can transform the implicit scheme in a "semi-implicit" scheme (also monotonous):

$$
S_{s_{1}, s_{2}}^{s e m i}(\rho, x, t, u)=t-\left(u(x)+\Delta \tau S_{s_{1}, s_{2}}^{i m p l}(\rho, x, u(x), u)\right),
$$



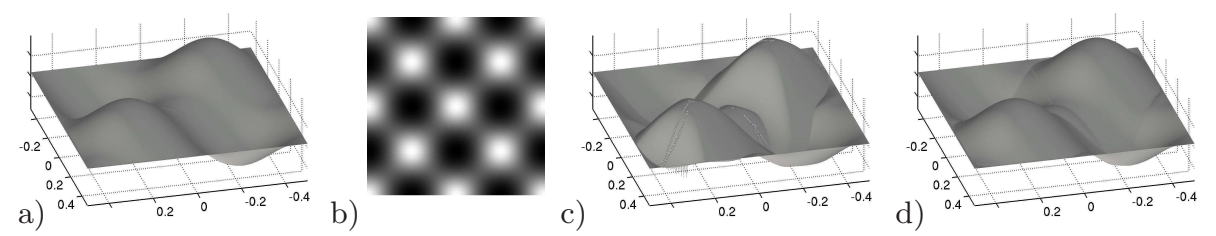

Fig. 4. a) original surface; b) image generated from a) by the Eikonal process [size $400 \times 400$ ]; c) reconstructed surface from b) after 15 iterations of Dupuis and Oliensis' algorithm (based on differential games) enforcing the exact Dirichlet condition on the boundary of the image and at all critical points: $\epsilon_{1}=0.015, \epsilon_{2}=5.7 e-05, \epsilon_{\infty}=0.35$; d) reconstructed surface by the implicit algorithm with the same boundary data and after the same number (15) of iterations as c): $\epsilon_{1}=0.002, \epsilon_{2}=1.0 e-05, \epsilon_{\infty}=0.014$.

where $\Delta \tau=\left(f_{g}\left(x, a_{0}\right) \cdot\left(1 / \Delta x_{1}, 1 / \Delta x_{2}\right)\right)^{-1} ; a_{0}$ being the optimal control. Let us emphasize that these two schemes have exactly the same solutions.

Using Barles and Souganidis definitions [2], we prove in [19] that these schemes are always monotonous and stable. Also, they are consistent with the genereric SFS equation as soon as the intensity image is Lipschitz continuous. Finally, when the Hamiltonian is coercive, we prove that the solutions of these schemes converge toward the unique SDVS of the "regularized" generic SFS equation, when $\rho \rightarrow 0$.

Remark: These two schemes have also a control interpretation. It is easy to verify that the implicit scheme is an extension of the control-based schemes proposed by $[23,15,22]$ and the semi-implicit scheme corresponds to the control-based scheme proposed by [6]. All these schemes have been designed for the "orthographic SFS" problem. Note that for a given Hamiltonian, they all have the same solutions. Therefore we have unified and generalized these various approaches.

Numerical algorithms for the generic SFS problem: In the previous section, we have proposed two schemes whose solutions $u^{\rho}$ converge toward the unique SDVS of the "regularized" generic SFS equation. For each scheme, we now describe an algorithm that computes an approximation of $u^{\rho}$.

For a fixed mesh size $\rho=\left(\Delta x_{1}, \Delta x_{2}\right)$, let us denote $x_{i j}:=\left(i \Delta x_{1}, j \Delta x_{2}\right)$ and $\mathcal{X}:=\left\{x_{i j} \in \Omega ; i, j \in \mathbb{Z}\right\}$. The algorithms consist of the following computation of the sequence of values $U_{i j}^{n}, n \geq 0\left(U_{i j}^{n}\right.$ being an approximation of $\left.u^{\rho}\left(x_{i j}\right)\right)$.

Algorithm 1 1. Initialisation $(n=0): U_{i j}^{0}=u_{0}\left(x_{i j}\right)$.

2. Choice of a pixel $x_{i j} \in \mathcal{X}$ and modification of $U_{i j}^{n}$ : We choose $U^{n+1}$ such that $\forall(k, l) \neq(i, j), U_{k l}^{n+1}=U_{k l}^{n} \quad$ and $S\left(\rho, x_{i j}, U_{i j}^{n+1}, U^{n}\right)=0$.

3. Choose the next pixel $x_{i j} \in \mathcal{X}$ in such a way that all pixels of $\mathcal{X}$ are regularly visited and go back to 2.

We prove in [19] that if $u_{0}$ is a subsolution or a supersolution, then the computed numerical approximations converge toward $u^{\rho}$. In their work, Rouy, Prados et al. $[23,15,22,20]$ use (some particular cases of) the implicit algorithm starting from a subsolution. When we start from a supersolution, we reduce the number of iterations by 3 orders of magnitude! In [20], Prados and Faugeras need around 

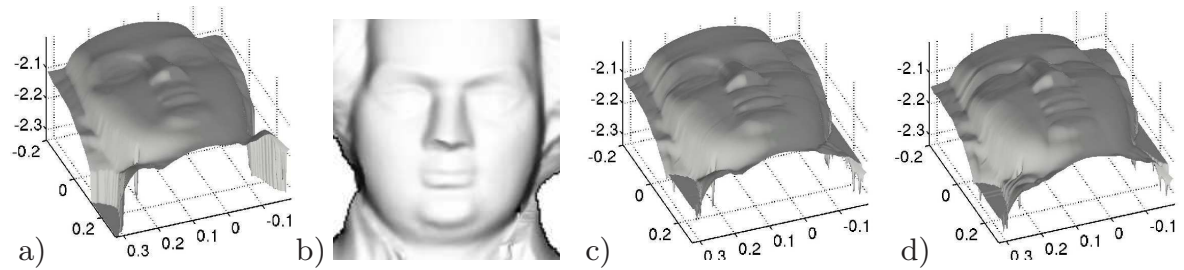

Fig. 5. a) original surface; b) image generated from a) [size $\simeq 200 \times 200$ ]; c) reconstructed surface from b) with the implicit algorithm (IA) after only 3 iterations, using the exact boundary data at all critical points and with state constraints on the boundary of the image: $\epsilon_{1} \simeq 0.58, \epsilon_{2} \simeq 0.0019, \epsilon_{\infty} \simeq 0.42 ;$ d) reconstructed surface by the IA (after 3 iterations) with state constraints on the boundary of the image and at all the critical points except at that on the nose: $\epsilon_{1} \simeq 0.60, \epsilon_{2} \simeq 0.0020, \epsilon_{\infty} \simeq 0.42$.

4000 iterations for computing the surface of the classical Mozart's face [29]. Starting from a supersolution (in practice, a large constant function $u_{0}$ does the trick!), only three iterations are sufficient for obtaining a good result; see figure 5. As an example, we show in figure 4 a comparison of our results with those of what we consider to be the most efficient algorithm of the SFS literature [6]. Figures 4-c) and 4-d) show the results returned by our implementation of this algorithm and our algorithm, respectively, after 15 iterations. The results are visually different. This visual difference is confirmed by the computation of the errors with respect to the original surface $\left(\epsilon_{1}, \epsilon_{2}\right.$ and $\epsilon_{\infty}$ are the errors of the computed surface measured according to the $L_{1}, L_{2}$ and $L_{\infty}$ norms, respectively). Nevertheless let us note that the cost of one update is slightly larger for our implicit algorithm than for the (semi-implicit) algorithm of Dupuis and Oliensis. This may also be because we have not optimized our code for this special case. Let us add that in this test, we have constrained the solution by the exact Dirichlet condition on the boundary of the image and at all the critical points. Let us recall that the SDVS method does not necessarily require boundary data. Figure 5 shows some reconstructions of the Mozart face when using the exact boundary data at all the critical points and state constraints on the boundary of the image (Fig.5-c), and with no boundary data, except for the tip of the nose (Fig.5-d). Moreover, let us emphasize that our implicit algorithm (as our semi-implicit one) allows to compute some numerical approximations of the SDVS of the degenerate (when the intensity reaches 1) and generic SFS problem. Thus, we only need to implement a single algorithm for all SFS modelizations. Finally, let us remark that, as the theory predicted, our algorithm shows an exceptional robustness to noise and errors on the parameters; This robustness is even bigger when we send the boundary to infinity (apply the state constraints). Figure 6 displays a reconstruction of Mozart's face from an image perturbed by additive uniformly distributed white noise (SNR $\simeq 5$ ) by using the implicit algorithm with the wrong parameters $\mathbf{l}_{\epsilon}=(0.2,-0.1)$ and $f_{\epsilon}=10.5$ (focal length) and without any boundary data. The original image Fig.6-a) has been synthetized with $\mathbf{l}=$ $(0.1,-0.3)$ and $f=3.5$. The angle between the initial light vector $\mathbf{L}$ and the corrupted light vector $\mathbf{L}_{\epsilon}$ is around $13^{\circ}$. More details, experimental comparisons 
and stability tests can be found in [19,21]. These reports also contain the proofs of all our statements.

a)

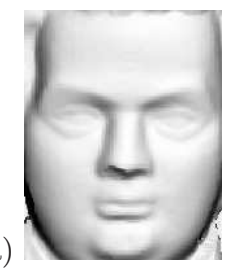

b)

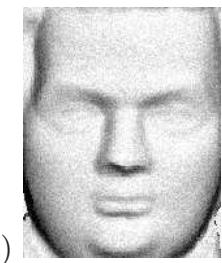

c)

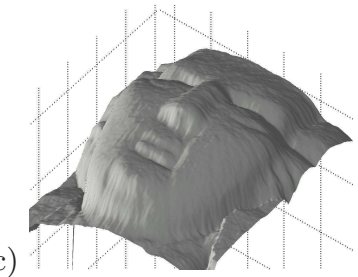

Fig. 6. a) Image generated from Mozart's face represented in Fig.5-a) with $\mathbf{l}=$ $(0.1,-0.3)$ and $f=3.5[$ size $\simeq 200 \times 200] ; \quad$ b) noisy image $(\mathrm{SNR} \simeq 5) ; \quad$ c) reconstructed surface from b) after 4 iterations of the implicit algorithm, using the incorrect parameters $\mathbf{l}_{\epsilon}=(0.2,-0.1)$ and $f_{\epsilon}=10.5$, and with state constraints on the boundary of the image and at all the critical points except at the critical point on the nose.

\section{Conclusion}

We have unified various formulations of the Lambertian SFS problem; in particular the orthographic and perspective problems. We have developed a new mathematical framework which unifies some SFS theories and generalizes them to all SFS Hamiltonians. Let us emphasize that we do not consider Mathematics as a goal in itself. Mathematics is simply a powerful tool allowing us to

- suggest some numerical methods and algorithms;

- certify algorithms, to guarantee their robustness and to describe their limitations;

- better understand what we compute. In particular, when the problem has several solutions, it allows to characterize all the solutions, a necessary preliminary step for deciding which solution we want to compute.

In effect, our theory ensures the stability and the convergence of our SFS method. Also it suggests a robust SFS algorithm which seems to be the most efficient iterative algorithm of the SFS literature. Moreover, our new class of weak solutions is really more adapted to the SFS specifications; in particular, it does not necessarily require boundary data. We are extending our approach to non Lambertian SFS and to SFS with discontinous images.

\section{References}

1. M. Bardi and I. Capuzzo-Dolcetta. Optimal control and viscosity solutions of Hamilton-Jacobi-Bellman equations. Birkhauser, 1997.

2. G. Barles and P. Souganidis. Convergence of approximation schemes for fully nonlinear second order equations. Asymptotic Analysis, 4:271-283, 1991.

3. F. Camilli and A. Siconolfi. Maximal subsolutions for a class of degenerate hamilton-jacobi problems. Indiana Univ. Math. J., 48(3):1111-1132, 1999. 
4. F. Camilli and A. Siconolfi. Nonconvex degenerate Hamilton-Jacobi equations. Mathematische Zeitschrift, 242:1-21, 2002.

5. F. Courteille, A. Crouzil, J.-D. Durou, and P. Gurdjos. Shape from Shading en conditions réalistes d'acquisition photographique. In Proceedings of RFIA'04, 2004.

6. P. Dupuis and J. Oliensis. An optimal control formulation and related numerical methods for a problem in shape reconstruction. The Annals of Applied Probability, 4(2):287-346, 1994.

7. J.-D. Durou and H. Maitre. On convergence in the methods of Strat and Smith for shape from shading. IJCV, 17(3):273-289, 1996.

8. M. Falcone, M. Sagona, and A. Seghini. A scheme for the shape-from-shading model with "black shadows". In Proceedings of ENUMATH 2001, 2001.

9. J. Hasegawa and C. Tozzi. Shape from shading with perspective projection and camera calibration. Computers and Graphics, 20(3):351-364, May 1996.

10. B. Horn and M. Brooks, editors. Shape from Shading. The MIT Press, 1989.

11. H. Ishii and M. Ramaswamy. Uniqueness results for a class of Hamilton-Jacobi equations with singular coefficients. Comm. Par. Diff. Eq., 20:2187-2213, 1995.

12. J. Kain and D. Ostrov. Numerical shape-from-shading for discontinuous photographic images. IJCV , 44(3):163-173, 2001.

13. K. Lee and C. Kuo. Shape from shading with perspective projection. CVGIP: Image Understanding, 59(2):202-212, 1994.

14. P.-L. Lions. Generalized Solutions of Hamilton-Jacobi Equations. Number 69 in Research Notes in Mathematics. Pitman Advanced Publishing Program, 1982.

15. P.-L. Lions, E. Rouy, and A. Tourin. Shape-from-shading, viscosity solutions and edges. Numer. Math., 64:323-353, 1993.

16. J. Oliensis. Uniqueness in shape from shading. IJCV, 2(6):75-104, 1991.

17. M. Penna. Local and semi-local shape from shading for a simple perspective image of a smooth object. CVGIP, 46:346-366, 1989.

18. E. Prados and O. Faugeras. Une approche du "Shape from Shading" par solutions de viscosité. Master's thesis, Université de Nice Sophia-Antipolis, France, 2001.

19. E. Prados and O. Faugeras. A mathematical and algorithmic study of the lambertian SFS problem for orthographic and pinhole cameras. Technical Report RR-5005, INRIA, Nov. 2003.

20. E. Prados and O. Faugeras. "Perspective Shape from Shading" and viscosity solutions. In Proceedings of ICCV'03, volume 2, pages 826-831, 2003.

21. E. Prados and O. Faugeras. A viscosity method for Shape from Shading without boudary data and some of its applications. Technical report, INRIA, To appear.

22. E. Prados, O. Faugeras, and E. Rouy. Shape from Shading and viscosity solutions. In Proceedings of ECCV'02, volume 2351, pages 790-804, May 2002.

23. E. Rouy and A. Tourin. A Viscosity Solutions Approach to Shape-from-Shading. SIAM Journal of Numerical Analysis, 29(3):867-884, June 1992.

24. H. M. Soner. Optimal control with state space constraints. SIAM J. Contr. Optim, 24:Part I: 552-562, Part II: 1110-1122, 1986.

25. P. Soravia. Optimal control with discontinuous running cost: eikonal equation and shape from shading. In 39th IEEE CDC, pages 79-84, 2000.

26. A. Tankus, N. Sochen, and Y. Yeshurun. A new perspective [on] Shape-fromShading. In Proceedings of ICCV'03, volume 2, pages 862-869, 2003.

27. I. Weiss. A perspective 3D formalism for shape from shading. In Proceedings of DARPA Image Understanding Workshop, volume 2, pages 1393-1402, May 1997.

28. S. Yuen, Y. Tsui, Y. Leung, and R. Chen. Fast marching method for sfs under perspective projection. In Proceeding of VIIP'02, pages 584-589, 2002.

29. R. Zhang, P.-S. Tsai, J.-E. Cryer, and M. Shah. Shape from Shading: A survey. IEEE Trans. PAMI, 21(8):690-706, Aug. 1999. 